\title{
ANPSORT II METHOD: AN EXPERIMENTATION TO ASSESS THE RESILIENCE OF A SOCIO-ECOLOGICAL SYSTEM.
}

\author{
VANESSA ASSUMMA, MARTA BOTTERO, ALESSIO ISHIZAKA
}

\begin{abstract}
In the current context of climate change, Decision Makers are ever more interested to use multidisciplinary and interdisciplinary approaches to support the practice of resilience thinking within long-term strategies. This paper presents an experimentation of a Multicriteria Decision Analysis approach (MCDA) developed through the Analytic Network Process Sorting II method (ANPSort II) to investigate the resilience of a SocioEcological Systems as a group of territorial clusters in the Grand-Est region, France. A set of indicators was defined to evaluate the resilience of territorial clusters, according to analyses performed through GIS and STEEP+SWOT Analysis. A survey was developed to investigate the importance of the set of indicators and to assess the resilience performance of the case study under investigation. The assessment was represented into a GIS spatial map to aid the visualization of the final classification. The ANPSort II method may be considered a very promising evaluation procedure to aid planners and Decision Makers to envision scenarios of territorial transformation for the planning of more sustainable and resilient cities and territories.
\end{abstract}

Keywords: Resilience, MCDA, ANPSort II.

\section{Introduction}

In recent years, planners and Decision Makers are more and more interested to incorporate resilience principles within territorial policy decisions to contrast the effects of climate change and guarantee a sustainable quality of life. Resilience is intended as the ability of a Socio-Ecological System (SES) to respond, to adapt and to transform itself in relation to stresses and disturbances, across space and time (Meerow et al., 2016). Several studies focused on the resilience conceptualization and many international documents and agendas stimulated the research to practice resilience thinking, even if few practices are still counted today. Evaluation procedures are moving to bridge this gap; within this context a very important role is played by the techniques of Multicriteria Decision Analysis (MCDA), which are ever more required by Decision Makers to solve complex SES's issues. In particular, sorting methods reveal particularly suitable when several alternatives must be evaluated and classified into homogeneous groups. The main novelty proposed in this paper is to investigate a recent extension of the sorting method the Analytic Network Process Sorting (ANPSort) (Ishizaka \& Pereira, 2020) into the Analytic Network Process Sorting II method (ANP Sort II) to assess the resilience performance of a SES: the Champagne-Ardenne landscape, France. This work intends to demonstrate the potentialities of the proposed approach to deal with complex decision problems. The model employs a set of resilience indicators which represent specific SES's dimensions. The final classification is represented into a GIS spatial map to better understand the results and subsequently to localize potential actions in the most critical areas. 
ISAHP Article: A Style Guide for Paper Proposals To Be Submitted to the International Symposium on the Analytic Hierarchy Process 2020, Web Conference.

\section{Literature Review}

Sorting methods allows to assign numerous alternatives into homogeneous classes, according to their performance. As far as the AHP/ANP methods are considered, their usefulness was explored in different disciplines (Table 1). Despite their multidisciplinary application, there is not yet a sorting method to assess resilience or climate change aspects.

Table 1. Selection of representative contributions on sorting methods.

\begin{tabular}{|l|l|l|}
\hline Author, Year & Description & Field \\
\hline $\begin{array}{l}\text { Ishizaka et al. } \\
(2012)\end{array}$ & $\begin{array}{l}\text { Development of the AHP Sort as new variant of the AHP } \\
\text { process to support decision problems of large scale. }\end{array}$ & Decision-making \\
\hline $\begin{array}{l}\text { Miccoli and } \\
\text { Ishizaka (2017) }\end{array}$ & $\begin{array}{l}\text { AHPSort II for a risk classification of municipalities to } \\
\text { wolf attack on livestock farms. }\end{array}$ & Risk Analysis \\
\hline $\begin{array}{l}\text { Ishizaka and } \\
\text { Pereira (2020) }\end{array}$ & $\begin{array}{l}\text { ANPSort method to provide a researcher classification in } \\
\text { the ambit of high education academy. }\end{array}$ & Education \\
\hline
\end{tabular}

\section{Hypotheses/Objectives}

The presented model aims to assess the resilience of a SES and support planners and decision-makers to define long-term strategies in the field of environmental assessment procedures of plans and programs.

\section{Research Design/Methodology}

The decision problem was structured according to the ANP Sort II method (Figure 1). It considered 5 clusters as specific SES's dimensions to explore its resilience (i.e. society, technology, environment, economy, and regional development). Each cluster is further divided into 3 nodes (or indicators) that represent the SES resilience properties, namely value, vulnerability, and coping capacity.

The indicators have been defined following the relevant literature and by means of specific analysis on the area through GIS and other tools, such as the STEEP+SWOT Analysis.

For example, the cluster Regional Development provides three indicators, i.e. Local Action Groups (LAGs) (no.), Land take index 1990-2018 (0; $\geq 1)$ and EIA and SEA procedures (no.) that represent the value, vulnerability and coping capacity of the Regional Development cluster, respectively. The database used for the data collection refers to European, national and regional data sources, e.g. INSEE, DREAL - Grand Est, or COPERNICUS. It provides the state of the art of a SES in the Grand-Est region (France), organized into 14 territorial clusters. For the development of the evaluation, a survey was proposed in September-October 2020 through a web-questionnaire to an expert of urban and territorial planning. The main steps of the ANP Sort II method are reported below.

1. Definition of qualitative classes $C_{i}, i=1, \ldots, 4$, which label the resilience performance as "poor", "medium", "good", and "strong";

2. Definition of local limiting profiles $\mathrm{lp}_{i j}$ or central profiles $\mathrm{cp}_{i j}$ with respect to the 4 classes. The limiting profile was chosen, intended as the minimum achievable performance of an indicator $j$ to belong to $C_{i}$ for an alternative $a_{k}$; 

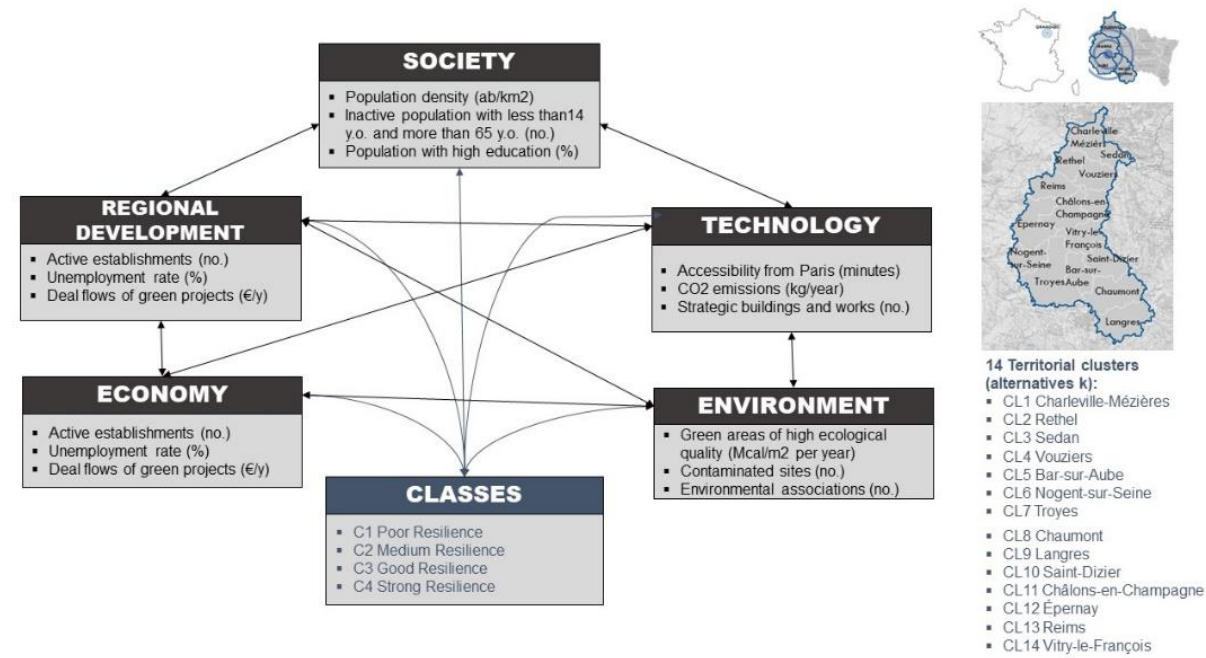

Figure 1. Structure of the decision problem (left) (Elaboration from Superdecisions) and geographical localization (right) (Elaboration on DIVA GIS data, 2018).

3. Influence matrix to identify the network's connections through the software Superdecisions;

4. The expert judges pairwise the network's elements, at nodes and clusters levels, on the Saaty's Scale. Superdecisions notifies incoherent judgements with the Consistency Ratio (CR must be $\leq 10 \%$ ) and allows to modify them. A set of weights is derived through the eigenvalue method;

5. The expert selects representative points $s_{o j}, o=1, \ldots, r p_{j}$ of each indicator;

6. The expert judges pairwise the $\mathrm{lp}_{i j}$ and $s_{o j}$ to obtain the priorities $\mathrm{p}_{i j}$ and $p_{o j}$;

7. Calculation of $\mathrm{p}_{k}$ and $\mathrm{lp}_{i}$ (see Eq.2, 3 and 4, Miccoli \& Ishizaka, 2017);

8. All influences detected between the network's elements, calculated through the eigenvalue method with the local priorities, are inserted within a supermatrix. Each column of the supermatrix is standardized to 1. Subsequently, the supermatrix is raised to power and then to a limiting power, where global priorities $\mathrm{p}_{k s}$ are provided;

9. Evaluation of global priorities $\mathrm{p}_{k}$ with $\mathrm{lp}_{i}$ to assign an alternative to a class;

10. Steps from 5 to 9 are replicated for each alternative of evaluation;

11. Refinement of those alternatives just above and below the $\operatorname{lp}_{i}$ with ANPSort. If both ANPSort and ANPSort II methods classifications are similar, the process is terminated. Otherwise, the alternatives must be further classified;

12. Elaboration of spatial maps to visualize the most resilient and the less resilient areas.

\section{Data/Model Analysis}

The expert was supported during the survey through a web-questionnaire by virtual conference platform and supplementary material, e.g. case study's dossier, STEEP+SWOT Analysis, or list of the indicators. The evaluation of the expert of urban and regional development is reported below. In the first section of the web-questionnaire, the expert answered to 5 groups of open questions to identify the local limiting profiles for each indicator, according to the resilience classes (Table 2). 
ISAHP Article: A Style Guide for Paper Proposals To Be Submitted to the International Symposium on the Analytic Hierarchy Process 2020, Web Conference.

Table 2. Limiting profiles of the indicator "Land take 1990-2018 (0; $\geq 1)$ " by DM5.

\begin{tabular}{|c|c|c|c|c|c|c|}
\hline Poor & $\mathrm{lp}_{i j}$ & Medium & $\mathrm{lp}_{i j}$ & Good & $\mathrm{lp}_{i j}$ & Strong \\
\hline & 0.30 & & 0.17 & & 0.10 & \\
\hline
\end{tabular}

In the second section, the expert compared pairwise the indicators at clusters and nodes levels, and these were then imported in the software Superdecisions (Table 3).

Table 3. Judgements in the cluster "Regional Development" for the class "Good resilience".

\begin{tabular}{|l|c|c|c|c|}
\hline Nodes (Indicators) & r.d.1 & r.d.2 & r.d.3 & Priorities \\
\hline r.d.1 Local Action Groups (no.) & 1 & 4 & $1 / 3$ & 0.27969 \\
\hline r.d.2 Land take 1990-2018 (0; $\geq 1$ ) & $1 / 4$ & 1 & $1 / 5$ & 0.09362 \\
\hline r.d.3 EIA and SEA procedures (no.) & 3 & 5 & 1 & 0.62670 \\
\hline \multicolumn{4}{|l|}{} & \multicolumn{3}{|l}{ CR 0.08247} \\
\hline
\end{tabular}

The priorities were elaborated through the unweighted, weighted and limit supermatrices, thus obtaining the limiting priorities. The limiting priorities of the indicators were normalized excluding the classes's priorities, thus obtaining $\mathrm{w}_{j}$.

In the third section, the expert selected some representative points with respect to the $\operatorname{lp}_{i j}$ previously identified. In the last section, both $\mathrm{lp}_{i j}$ and $s_{o j}$ were compared pairwise to obtain the local priorities $\mathrm{p}_{k j}$ (Figure 2).

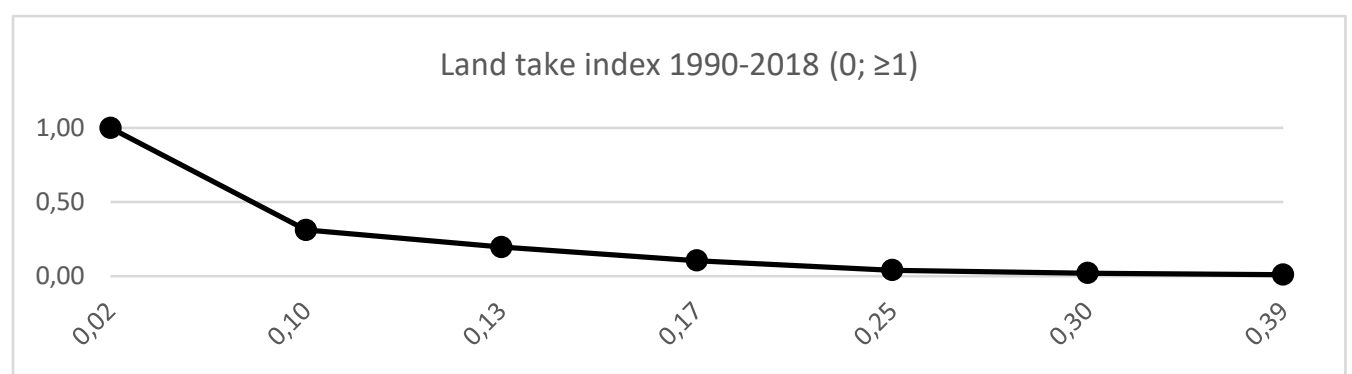

Figure 2. "Land take index" function based on limiting profiles and representative points.

Following the steps 10 to 12 , the expert's classification was obtained for the 14 territorial clusters. A final tuning was performed with the expert on selected territorial clusters. The ANPSort classifications provided similarity, with exception for the CL2 Rethel and CL12 Épernay (AHPSort= Good resilience; AHPSort II = Medium resilience) and CL13 Reims (AHPSort $=$ Good resilience; AHPSort II = Strong resilience). The final classification was represented into a GIS-based spatial map (Figure 3, see Appendix).

\section{Limitations}

The set of indicators considered only some aspects of a complex phenomenon. The expert had some difficulty to identify the limiting profiles due to the lack of a threshold value at national or regional level.

\section{Conclusions}


The paper demonstrated the potentialities of ANPSort II to assess the resilience of a SESDue to the multidisciplinary nature of the problem under investigation, other experts have been engaged into the ongoing sorting process and the results will be shared within the panel in order to have a common vision on the final classification. Despite it represents an experimentation, we retain ANPSort II able to support planners and Decision Makers in the planning of scenarios of transformation. The application to a real case study raised some future perspectives. A threshold values will be considered to aid the experts in the identification of limiting or central profiles. The ANPSort II obtained by the experts' evaluations will be grouped together. The model will be replicated by involving real local actors and stakeholders to define a protocol of actions to increase the resilience of the case study under investigation.

\section{Key References}

Meerow, S., Newell, J. P., Stults, M. (2016). Defining urban resilience: a review. Landscape and Urban Planning, 147, 38-49. DOI: 10.1016/j.landurbplan.2015.11.011

Ishizaka, A. Nemery, P., Pearman, C. (2012). AHPSort: an AHP based method for sorting problems. International Journal of Production Research, 50(17), 4767-4784. DOI: 10.1080/00207543.2012.657966

Ishizaka, A., \& Pereira, V. (2020). Portraying an employee performance management system based on multi-criteria decision analysis and visual techniques. International Journal of Manpower, 37(4), 628-659. DOI:10.1108/IJM-07-2014-0149

Miccoli, F. \&, Ishizaka, A. (2017). Sorting municipalities in Umbria according to the risk of wolf attacks with AHPSort II. Ecological Indicators, 73, 741-755. DOI:10.1016/j.ecolind.2016.10.034

\section{Appendix}

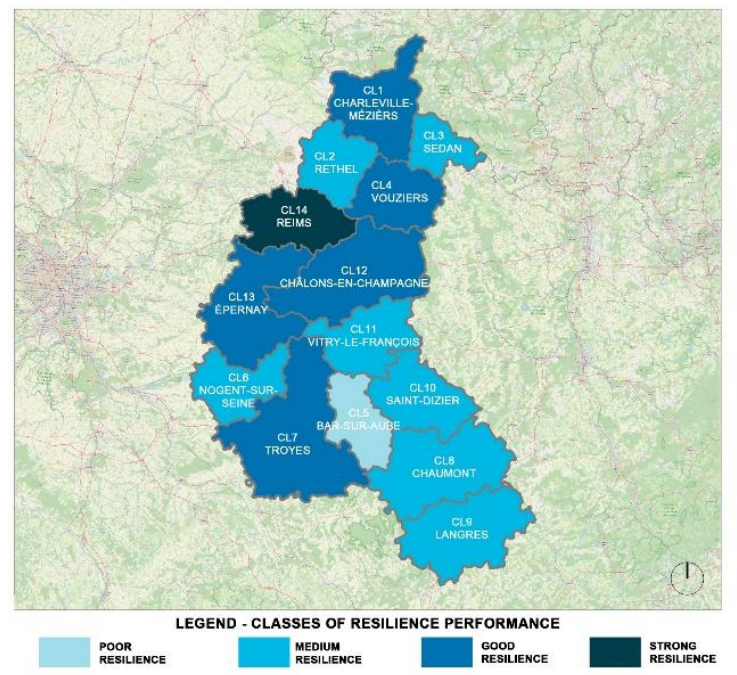

Figure 3. Final classification of the expert on the resilience performance.

International Symposium on the Analytic Hierarchy Process
DEC. 3 - DEC. 6, 2020 